\title{
Agrupamento de paises segundo indicadores de padrão de vida
}

\section{Clustering of countries evaluated by standard of living indicators}

\section{Paulo Roberto Curi*}

\begin{abstract}
CURI, P. R. Agrupamento de países segundo indicadores de padrão de vida. Rev. Saúde Pública, 27: 127-34, 1993. Foram estudados 125 países avaliados por um conjunto de 26 indicadores básicos, de saúde, econômicos e educacionais, usando-se três métodos estatísticos multivariados: Análise de Agrupamento, Análise de Componentes Principais e Análise de Variância Multivariada. As variáveis mais discriminatórias foram a expectativa de vida, as taxas de mortalidade infantil e de menores de cinco anos, as taxas de natalidade e de fertilidade e a taxa de matrícula no segundo grau para o sexo feminino. Os países foram ordenados de acordo com um "índice de padrão de vida" e separados em cinco grupos.
\end{abstract}

Descritores: Qualidade de vida. Análise por conglomerados. Análise discriminante. Estatísticas de saúde.

\section{Introduçăo}

Diversas instituições entre as quais o Banco Mundial e a Organização das Naçðes Unidas se dedicam à publicação anual de indicadores econômicos, demográficos, de saúde, culturais e educacionais dos países. Estas informaçőes, na maioria das vezes, têm sido utilizadas sem um enfoque comparativo abrangente e acabam servindo como elementos ilustrativos de reportagens publicadas em revistas não-científicas. Mesmo 0 informe "Situação Mundial da Infância - 1991"6, do qual foram extraídos muitos dos dados utilizados no presente artigo, faz uso parcial dessas estatísticas o que pode, em parte, ser creditado à dificuldade de seu manuseio face ao volume de dados nele inserido.

Visando modificar esta situação, tem sido proposta a utilização de métodos de análise multivariada que permitem explorar melhor essas tabelas de dados onde os países são caracterizados por conjuntos de variáveis 23,4 .

No presente artigo foram estudados 125 países, cujo critério de inclusão foi dependente da disponibilidade de informação para um conjunto de 26 indicadores sociais, econômicos, demográficos, de saúde, culturais e educacionais

\footnotetext{
* Serviço de Estatística e Computação da Faculdade de Medicina Veterinária e Zootecnia da UNESP - Botucatu, SP - Brasil.
}

Separatas/Reprints: P. R. Curi - Rubião Júnior - Campus Universitário - 18618-000 - Botucatu, SP Brasil. que possibilitassem uma visão ampla do padrão de vida dos mesmos.

O principal objetivo do presente artigo é mostrar a utilização de métodos de análise multivariada para discutir a capacidade discriminatória dos indicadores usados, no sentido de separar o conjunto dos paises em grupos cujas caracteristicas desejadas são a homogeneidade interna de seus componentes, a heterogeneidade entre membros de diferentes grupos e a caracterização dos grupos resultantes para o conjunto dos indicadores.

Não se pretendeu explicar exaustivamente os resultados do ponto de vista da determinação social do processo traduzido pelos indicadores utilizados.

\section{Materlal e Método}

Dentre as inúmeras variáveis que poderiam ser utilizadas, as escolhidas preencheram alguns critérios de seleção, tais como, disponibilidade da informação para a maioria dos países e importância à priori como indicadora do padrăo de vida. As siglas de identificação e as definiçōes das 26 variáveis usadas são apresentadas a seguir. Os dados encontram-se publicados ${ }^{6,7}$, não sendo aqui transcritos.

EXP: expectativa de vida ao nascer, ALF: taxa de alfabetização de adultos; NAT: taxa de natalidade; FERT: taxa de fertilidade; LEIT, MED, ENF: número de habitantes por leito hospitalar, por médico e por enfermeiro, respectivamente; TMM5: taxa de mortalidade de menores de 5 anos; TMI: taxa de mortalidade infantil; RNBP: 
percentagem de recém-nascidos com baixo peso ao nascer; CAL: suprimento calórico diário per capita como percentagem da necessidade; DPT, POL, SAR: percentagem de crianças com 1 ano imunizadas, respectivamente, com vacinas tríplice, para poliomielite e para sarampo; NAS: percentagem dos nascimentos (partos) realizados por pessoal especializado; MAT: taxa de mortalidade materna por causas relacionadas a gestação para cada 100.000 nascidos vivos; PNB: produto nacional bruto em dólares; M1G: taxa bruta de matrícula no primeiro grau escolar; RAD (TV): número de aparelhos de rádio (televisão) disponíveis por 1.000 habitantes; $\mathrm{ClG}$ : percentagem de matriculados na primeira série que concluem o primeiro grau; M2GM (M2GF): taxa de matrícula no segundo grau para o sexo masculino (feminino); CPOP: taxa de crescimento populacional; URB: taxa de população urbana; CURB: taxa de crescimento da população urbana. Maiores detalhes e explicaçōes sobre as variáveis podem ser vistos em publicação da UNICEF'.

Outros indicadores que, pela sua importância, poderiam ser incluídos no estudo, não o foram em virtude de não estarem disponíveis para grande número de países. Pela mesma razão alguns países não fizeram parte do estudo. A relação dos 125 países, com seus respectivos números de identificação, encontra-se na Tabela 2.

Como as variáveis não apresentam a mesma escala (e/ou unidade) foi utilizada uma transformação de modo a torná-las adimensionais e com variação no intervalo de 0 a 1 .

Para a análise estatística foram empregados três métodos multivariados apresentados, discutidos e exemplificados, entre outros, por Sneath e Solal ${ }^{5}$ e Curi' ${ }^{1}$ :

a) Análise de Agrupamento (AA) utilizada calculando-se a Distância Euclidiana Média entre os países, com o objetivo de separá-los em grupos (conglomerados ou "clusters") com o uso do algorítmo UPGMA. O resultado final da AA é um gráfico, denominado dendrograma, que auxilia a definir os agrupamentos de países similares para o conjunto das 26 variáveis;

b) Análise de Componentes Principais (ACP) utilizada primeiro para reduzir o conjunto das 26 variáveis originais correlacionadas a um conjunto de novas variáveis não correlacionadas que são os componentes principais. Cada componente principal $(\mathrm{Y})$ é uma combinação linear das variáveis originais. Quando os dois primeiros componentes principais $\left(Y_{1}\right.$ e $\left.Y_{2}\right)$ conseguem reter cumulativamente uma quantidade suficiente da informação total contida no conjunto das variáveis originais, cada país, que era definido pelas 26 variáveis, pode ser definido pelas duas novas variáveis $\left(\mathrm{Y}_{1}\right.$ e $\left.\mathrm{Y}_{2}\right) \circ$ que possibilita sua localização como um ponto em um gráfico bidimensional. A Figura resultante neste processo, analisada em conjunto com 0 dendrograma da AA, permite inferir os agrupamentos de países. Os grupos devem se caracterizar pela maior homogeneidade interna de suas unidades e pela heterogeneidade entre unidades de diferentes grupos. A ACP possibilita, ainda, discutir a capacidade discriminatória das variáveis no processo de formação dos grupos o que $\varepsilon$ indicado pelo coeficiente de correlação ("peso") da variável na combinação linear que resultou o componente principal (quanto maior o valor absoluto do coeficiente de correlação maior o "peso"). A interpretação da combinação linear que forma o componente principal, em especial o primeiro $\left(Y_{1}\right)$, pode fornecer informação prática fundamental na análise dos resultados obtidos;

c) Análise de Variância Multivariada (MAV) utilizada com o objetivo de verificar se os agrupamentos de países resultantes com o emprego da ACP e da AA diferiram significativamente (heterogeneidade entre grupos) e para quais variáveis estas diferenças se verificaram. Possibilita, ainda, verificar a adequação de cada país ao seu respectivo grupo o que é feito por um processo probabilístico de reclassificação dos paises, com o uso de funções driscriminantes lineares, geradas no processo. Quanto menores resultarem as taxas de erro de reclassificação, mais coerentes serão os agrupamentos propostos (homogeneidade dentro dos grupos). Como resultado final desta análise, os países podem ser representados em gráfico utilizando duas variáveis canônicas (CAN1, CAN2) geradas no processo.

Portanto, a análise foi iniciada com um conjunto de 125 paises caracterizados por 26 indicadores de seu padrão de vida, essa informação foi resumida a duas novas variáveis não correlacionadas, os países foram separados em grupos caracterizados pela homogeneidade interna e heterogeneidade externa, foi verificado o poder discriminatório das variáveis no processo de separação dos grupos e foi discutida a adequação da classificação de cada pais no respectivo grupo.

\section{Resultados e Discussåo}

A Tabela 1 apresenta os coeficientes de correlação entre as variáveis originais e os dois componentes principais $\left(Y_{1}\right.$ e $\left.Y_{2}\right)$, bem como a orde- 
Tabela 1. Coeficiente de correlação entre as variáveis originais $\theta$ os dois primeiros componentes principais $\left(Y_{1} \ominus Y_{2}\right)$. Percentagem da informação contida nos 26 indicadores $\theta$ retida em cada componente principal (\% INFORM.). Ordenação das variáveis originais de acordo com sua capacidade discriminatória (ordem).

\begin{tabular}{lccc}
\hline Correlação* & $Y_{1}$ & $Y_{2}$ & Ordem \\
\hline EXP & 0,965 & $-0,096$ & 1 \\
ALF & 0,895 & $-0,052$ & 9 \\
NAT & $-0,936$ & $-0,163$ & 3 \\
FERT & $-0,910$ & $-0,177$ & 5 \\
LEIT & $-0,558$ & 0,077 & 25 \\
MED & $-0,679$ & 0,271 & 22 \\
ENF & $-0,493$ & 0,030 & 26 \\
TMM5 & $-0,910$ & 0,160 & 6 \\
TMI & $-0,953$ & 0,133 & 2 \\
RNBP & $-0,692$ & 0,031 & 24 \\
CAL & 0,829 & 0,013 & 10 \\
DPT & 0,704 & $-0,566$ & 16 \\
POL & 0,736 & $-0,550$ & 14 \\
SAR & 0,637 & $-0,609$ & 23 \\
NAS & 0,897 & $-0,063$ & 8 \\
MAT & $-0,762$ & 0,258 & 15 \\
PNB & 0,685 & 0,366 & 21 \\
M1G & 0,697 & $-0,337$ & 19 \\
RAD & 0,695 & 0,322 & 20 \\
TV & 0,781 & 0,281 & 13 \\
C1G & 0,756 & 0,056 & 17 \\
M2GM & 0,905 & 0,092 & 7 \\
M2GF & 0,943 & 0,101 & 4 \\
CPOP & $-0,697$ & $-0,502$ & 18 \\
URB & 0,803 & 0,108 & 12 \\
CURB & $-0,781$ & $-0,390$ & 11 \\
\hline \% Inform. & 68 & 8 & \\
\% Inform. Acum. & 68 & 76 & \\
\hline & 0.39 & \\
\hline
\end{tabular}

Fonte: UNICEF6 (19\&1); World Bank7 (1191)

- Significado das siglas: ver texto,?.

nacão das variáveis conforme sua capacidade discriminatória. As variáveis mais discriminatórias foram: EXP, TMI, NAT, M2GF, FERT e TMM5 enquanto as menos foram: ENF, LEIT, RNBP, SAR e MED.

As variáveis, à priori, podem ser separadas em duas classes: aquelas cujos valores elevados indicam melhor padrão de vida, referidas como sendo de classe 1 (EXP, ALF, CAL, DPT, POL, SAR, NAS, PNB, M1G, RAD, TV, C1G, M2GM, M2GF e URB) e as restantes, cujos valores elevados indicam padrão inferior, referidas como sendo de classe 2.

Pela Tabela 1 pode ser visto que $Y_{1}$ foi positivamente correlacionado com as variáveis de classe 1, o contrário ocorrendo com as de classe 2 . Assim sendo, quanto melhores forem os indicadores do país maior será seu respectivo $Y_{1}$. Portanto, o primeiro componente principal, que reteve $68 \%$ da informação contida no conjunto das 26 variáveis originais, pode ser considerado como um "índice de padrão de vida" do país.
O segundo componente $\left(\mathrm{Y}_{2}\right)$ apresentou maiores correlaçōes positivas com PNB, RAD e TV e negativas com SAR, POL, DPT e CPOP. Os paises com $Y_{2}$ grande apresentaram ou pequenas taxas de imunização e crescimento populacional ou valores elevados de PNB, RAD e TV. Como $\mathrm{Y}_{2}$ reteve $8 \%$ da informação contida nas variáveis originais, seu significado prático mereceu pouco destaque. Os menores valores de $\mathrm{Y}_{2}$ ocorreram para Tanzânia (96), Botswana (69) (países com taxa de imunização maiores que 80\%) e Omã (63) que associou altas taxas de imunização (maiores que $90 \%$ ) com elevado PNB.

$\mathrm{Na}$ Tabela 2 os países estão listados em ordem decrescente de "padrão de vida", conforme indicado pelo valor de $Y_{1}$. A Figura 1 construída utilizando-se os pares ordenados $\left(\mathrm{Y}_{1}\right.$ e $\left.\mathrm{Y}_{2}\right)$ de cada país, obtidos na ACP, e a Figura 2, representando o dendrograma da AA, possibilitaram inferir a formação de 5 grupos de países. Na Tabela 2 os grupos estão separados por linhas horizontais e nas Figuras 1 e 2 estão delimitados. Pela análise conjunta das duas figuras verificou-se que, para dois países, não houve concordância: o Iêmen (58) foi classificado em G5 na Figura 1 e em G4 na Figura 2, e a Espanha (116) foi classificada em G1 na Figura 1 e em G2 na Figura 2. As demais classificaçōes foram concordantes nas duas análises. Como a ACP é um procedimento estatístico mais rigoroso em seus aspectos matemáticos, optou-se pelos 5 agrupamentos por ela fornecidos.

Em G5 localizaram-se os países com os piores indicadores (menores valores de $Y_{1}$, com média igual a $-0,124$ e desvio padrão de 0,013 para este componente). $\mathrm{O}$ grupo mostrou-se pouco homogêneo quanto aos valores de $Y_{2}$, destacando-se países com os maiores valores para este componente (Afeganistão (30), Guiné (79), Chade (73), Somália (94), Nigéria (87) e Guiné-Bissau (78)), provavelmente devido a suas baixas taxas de imunização (DPT, POL e SAR entre 15 e $30 \%$ ).

O G4 foi o mais heterogêneo dos grupos com relação aos valores de $\mathrm{Y}_{1}$ (média de 0,062 e desvio padrão de 0,025 ), sendo constituído por 37 países, em sua maioria africanos.

O grupo intermediário (G3) formado por 33 países, entre os quais se incluiu o Brasil (1) foi dos menos homogêneos (junto com G4) com valor médio de $Y_{1}$ igual a 0,023 e desvio padrão de 0,021 . O Líbano (62) e Omã (63) foram os países menos caracteristicos do grupo, o que pode ser verificado nas Figuras 1 e 2.

O G2, com 19 países, mostrou-se bastante homogêneo, principalmente para valores de $Y_{1}$ (média igual a 0,084 com desvio de 0,009 ).

O grupo dos países com melhores indicadores (G1) resultou bastante homogêneo tanto para va- 
Tabela 2. Ordenação decrescente dos países quanto ao "padrão de vida" indicado por $Y_{1}$, nome (número de identificaçāo do país) e valores dos 2 primeiros componentes principais $\left(Y_{1} \in Y_{2}\right)$. Separação em 5 grupos (G1 a G5).

\begin{tabular}{|c|c|c|c|c|c|c|c|c|c|c|c|}
\hline & Ordem & Pais & (Ne) & $Y_{1}$ & $Y_{2}$ & Ordem & Pais & $\left(N^{2}\right)$ & $Y_{1}$ & $Y_{2}$ & \\
\hline \multirow[b]{2}{*}{$\overline{0}$} & \multirow{2}{*}{$\begin{array}{r}1 \\
2 \\
3 \\
4 \\
5 \\
6 \\
7 \\
8 \\
9 \\
10 \\
11 \\
12 \\
13 \\
14 \\
15 \\
16 \\
17 \\
18\end{array}$} & \multirow{2}{*}{$\begin{array}{l}\text { EUA } \\
\text { Dinamarca } \\
\text { Holanda } \\
\text { Finlândia } \\
\text { Japão } \\
\text { Suécia } \\
\text { Suiça } \\
\text { Canadá } \\
\text { Alemannha } \\
\text { Bélgica } \\
\text { Austrália } \\
\text { Noruega } \\
\text { França } \\
\text { R. Unido } \\
\text { Espanha } \\
\text { Austria } \\
\text { Itália } \\
\text { N. Zelândia }\end{array}$} & \multirow{2}{*}{$\begin{array}{l}(024) \\
(103) \\
(113) \\
(104) \\
(028) \\
(106) \\
(111) \\
(023) \\
(110) \\
(112) \\
(025) \\
(105) \\
(117) \\
(108) \\
(116) \\
(109) \\
(118) \\
(026)\end{array}$} & \multirow{2}{*}{$\begin{array}{l}0,136 \\
0,134 \\
0,129 \\
0,128 \\
0,127 \\
0,125 \\
0,125 \\
0,124 \\
0,124 \\
0,119 \\
0,119 \\
0,118 \\
0,116 \\
0,115 \\
0,110 \\
0,107 \\
0,104 \\
0,100\end{array}$} & \multirow{2}{*}{$\begin{array}{l}0,033 \\
0,032 \\
0,022 \\
0,031 \\
0,038 \\
0,024 \\
0,029 \\
0,031 \\
0,041 \\
0,034 \\
0,017 \\
0,034 \\
0,031 \\
0,037 \\
0,028 \\
0,037 \\
0,044 \\
0,036\end{array}$} & $\begin{array}{l}64 \\
65 \\
66 \\
67 \\
68 \\
69 \\
70\end{array}$ & $\begin{array}{l}\text { Síria } \\
\text { Líbano } \\
\text { Equador } \\
\text { Indonésia } \\
\text { Omã } \\
\text { Argélia } \\
\text { Iraque }\end{array}$ & $\begin{array}{l}(064) \\
(052) \\
(006) \\
(037) \\
(063) \\
(050) \\
(060)\end{array}$ & $\begin{array}{r}0,002 \\
0,002 \\
0,001 \\
-0,002 \\
-0,004 \\
-0,011 \\
-0,016\end{array}$ & $\begin{array}{l}-0,043 \\
-0,029 \\
-0,004 \\
-0,022 \\
-0,050 \\
-0,024 \\
-0,018\end{array}$ & 8 \\
\hline & & & & & & \multirow{3}{*}{$\begin{array}{l}71 \\
72 \\
73 \\
74 \\
75 \\
76 \\
77 \\
78 \\
79 \\
80 \\
81 \\
82 \\
83 \\
84 \\
85 \\
86 \\
87 \\
88 \\
89 \\
90 \\
91 \\
92 \\
93 \\
94 \\
95 \\
96 \\
97 \\
98 \\
99 \\
100 \\
101 \\
102 \\
103 \\
104 \\
105 \\
106 \\
107\end{array}$} & \multirow{3}{*}{$\begin{array}{l}\text { Paraguai } \\
\text { El Salvador } \\
\text { Honduras } \\
\text { Africa do Sul } \\
\text { Zimbabue } \\
\text { Nicarágua } \\
\text { Botswana } \\
\text { Marrocos } \\
\text { India } \\
\text { Gabāo } \\
\text { Zàmbia } \\
\text { Guatemala } \\
\text { Bolivia } \\
\text { Congo } \\
\text { Gana } \\
\text { R. C. Afric. } \\
\text { Lesoto } \\
\text { Quênia } \\
\text { Tanzânia } \\
\text { Haitj } \\
\text { Paquistão } \\
\text { Senegal } \\
\text { Zaire } \\
\text { Camarōes } \\
\text { Sudão } \\
\text { Madagascar } \\
\text { Benim } \\
\text { Namíbia } \\
\text { Togo } \\
\text { Uganda } \\
\text { Camboja } \\
\text { Nigéria } \\
\text { C. Marfim } \\
\text { Libéria } \\
\text { Burundi } \\
\text { S. Leoa } \\
\text { Ruanda }\end{array}$} & \multirow{3}{*}{$\begin{array}{l}(007) \\
(013) \\
(015) \\
(102) \\
(101) \\
(017) \\
(069) \\
(053) \\
(032) \\
(076) \\
(100) \\
(021) \\
(003) \\
(074) \\
(077) \\
(090) \\
(080) \\
(089) \\
(096) \\
(014) \\
(033) \\
(092) \\
(099) \\
(072) \\
(095) \\
(082) \\
(068) \\
(086) \\
(097) \\
(098) \\
(047) \\
(088) \\
(075) \\
(081) \\
(071) \\
(093) \\
(091)\end{array}$} & \multirow{3}{*}{$\begin{array}{l}-0,018 \\
-0,019 \\
-0,021 \\
-0,021 \\
-0,023 \\
-0,027 \\
-0,028 \\
-0,033 \\
-0,039 \\
-0,046 \\
-0,049 \\
-0,049 \\
-0,050 \\
-0,054 \\
-0,059 \\
-0,064 \\
-0,065 \\
-0,068 \\
-0,072 \\
-0,072 \\
-0,072 \\
-0,073 \\
-0,074 \\
-0,074 \\
-0,075 \\
-0,075 \\
-0,080 \\
-0,082 \\
-0,082 \\
-0,083 \\
-0,084 \\
-0,084 \\
-0,089 \\
-0,089 \\
0,091 \\
-0,098 \\
-0,102\end{array}$} & \multirow{3}{*}{$\begin{array}{l}-0,024 \\
-0,002 \\
-0,041 \\
0,006 \\
-0,027 \\
-0,025 \\
-0,055 \\
-0,004 \\
-0,022 \\
-0,027 \\
-0,039 \\
-0,012 \\
0,020 \\
-0,018 \\
0,025 \\
-0,020 \\
-0,025 \\
-0,032 \\
-0,051 \\
0,032 \\
-0,041 \\
0,006 \\
0,028 \\
-0,003 \\
0,005 \\
0,010 \\
-0,012 \\
0,005 \\
-0,007 \\
-0,030 \\
0,038 \\
0,002 \\
0,008 \\
0,026 \\
-0,018 \\
-0,007 \\
-0,042\end{array}$} & \multirow{3}{*}{ 思 } \\
\hline$\widetilde{్}$ & $\begin{array}{l}19 \\
20 \\
21 \\
22 \\
23 \\
24 \\
25 \\
26 \\
27 \\
28 \\
29 \\
30 \\
31 \\
32 \\
33 \\
34 \\
35 \\
36 \\
37\end{array}$ & $\begin{array}{l}\text { Grécia } \\
\text { Cingapura } \\
\text { Irlanda } \\
\text { Hungria } \\
\text { Cuba } \\
\text { Bulgária } \\
\text { Israel } \\
\text { Polônia } \\
\text { Rep. Coréia } \\
\text { Tchecoslov. } \\
\text { Uruguai } \\
\text { R.Dem. Coréia } \\
\text { Romênia } \\
\text { Argentina } \\
\text { URSS } \\
\text { Chile } \\
\text { lugoslávia } \\
\text { Tr-Tobago } \\
\text { Portugal }\end{array}$ & $\begin{array}{l}(115) \\
(035) \\
(107) \\
(121) \\
(012) \\
(120) \\
(048) \\
(123) \\
(042) \\
(125) \\
(009) \\
(043) \\
(124) \\
(002) \\
(029) \\
(004) \\
(122) \\
(020) \\
(114)\end{array}$ & $\begin{array}{l}0,097 \\
0,096 \\
0,095 \\
0,095 \\
0,094 \\
0,093 \\
0,092 \\
0,088 \\
0,083 \\
0,082 \\
0,082 \\
0,080 \\
0,078 \\
0,077 \\
0,077 \\
0,076 \\
0,076 \\
0,068 \\
0,068\end{array}$ & $\begin{array}{r}0,008 \\
0,009 \\
0,025 \\
0,003 \\
-0,006 \\
-0,007 \\
-0,001 \\
-0,007 \\
0,009 \\
-0,003 \\
0,006 \\
-0,025 \\
0,002 \\
-0,005 \\
0,020 \\
-0,021 \\
-0,006 \\
0,000 \\
-0,013\end{array}$ & & & & & & \\
\hline \multirow{2}{*}{\multicolumn{2}{|c|}{$\begin{array}{l}38 \\
39 \\
40 \\
41 \\
42 \\
43 \\
44 \\
45 \\
46 \\
47 \\
48 \\
49 \\
50 \\
51 \\
52 \\
53 \\
54 \\
55 \\
56 \\
57 \\
58 \\
59 \\
60 \\
61 \\
62 \\
63\end{array}$}} & \multirow{2}{*}{$\begin{array}{l}\text { Jamaica } \\
\text { Albânia } \\
\text { Kwait } \\
\text { Libano } \\
\text { Panamá } \\
\text { Sri Lanca } \\
\text { Em. Ar. Un. } \\
\text { Costa Rica } \\
\text { México } \\
\text { Malásia } \\
\text { China } \\
\text { Jordânia } \\
\text { Colômbia } \\
\text { Mongólia } \\
\text { Venezuela } \\
\text { Tailândia } \\
\text { Brasil } \\
\text { Filipinas } \\
\text { Turquia } \\
\text { Tunísia } \\
\text { Peru } \\
\text { Irã } \\
\text { Vietnã } \\
\text { Egito } \\
\text { Rep. Domin. } \\
\text { A. Saúdita }\end{array}$} & \multirow{2}{*}{$\begin{array}{l}(016) \\
(119) \\
(056) \\
(062) \\
(018) \\
(044) \\
(057) \\
(011) \\
(022) \\
(039) \\
(027) \\
(061) \\
(005) \\
(040) \\
(010) \\
(045) \\
(001) \\
(036) \\
(049) \\
(054) \\
(008) \\
(059) \\
(046) \\
(051) \\
(019) \\
(055)\end{array}$} & \multirow{2}{*}{$\begin{array}{l}0,058 \\
0,057 \\
0,055 \\
0,054 \\
0,049 \\
0,048 \\
0,040 \\
0,040 \\
0,038 \\
0,037 \\
0,036 \\
0,034 \\
0,034 \\
0,031 \\
0,029 \\
0,018 \\
0,018 \\
0,017 \\
0,016 \\
0,016 \\
0,016 \\
0,011 \\
0,011 \\
0,009 \\
0,003 \\
0,003\end{array}$} & \multirow{2}{*}{$\begin{array}{r}-0,007 \\
-0,027 \\
-0,020 \\
0,028 \\
-0,024 \\
-0,014 \\
-0,023 \\
-0,040 \\
-0,015 \\
-0,032 \\
-0,042 \\
-0,037 \\
-0,020 \\
-0,024 \\
0,001 \\
-0,025 \\
-0,015 \\
-0,028 \\
-0,009 \\
-0,028 \\
0,002 \\
-0,039 \\
-0,030 \\
-0,015 \\
-0,025 \\
-0,041\end{array}$} & & & & & & \\
\hline & & & & & & $\begin{array}{l}108 \\
109 \\
110 \\
111 \\
112 \\
113 \\
114 \\
115 \\
116 \\
117 \\
118 \\
119 \\
120 \\
121 \\
122 \\
123 \\
124 \\
125\end{array}$ & $\begin{array}{l}\text { Laos } \\
\text { Butão } \\
\text { lemen } \\
\text { Mauritânia } \\
\text { Nepal } \\
\text { Malaui } \\
\text { Bangladexe } \\
\text { Chade } \\
\text { Guiné-Bissau } \\
\text { Etiópia } \\
\text { Afeganistāo } \\
\text { Burqu. -Faso } \\
\text { Angola } \\
\text { Moçambique } \\
\text { Niger } \\
\text { Guiné } \\
\text { Somália } \\
\text { Mali }\end{array}$ & $\begin{array}{l}(038) \\
(034) \\
(058) \\
(066) \\
(041) \\
(083) \\
(031) \\
(073) \\
(078) \\
(065) \\
(030) \\
(070) \\
(067) \\
(085) \\
(087) \\
(079) \\
(094) \\
(084)\end{array}$ & $\begin{array}{l}-0,104 \\
-0,106 \\
-0,107 \\
-0,108 \\
-0,110 \\
-0,114 \\
-0,117 \\
-0,121 \\
-0,127 \\
-0,128 \\
-0,129 \\
-0,129 \\
-0,131 \\
-0,132 \\
-0,134 \\
-0,142 \\
-0,142 \\
-0,143\end{array}$ & $\begin{array}{r}0,039 \\
-0,011 \\
-0,005 \\
0,038 \\
-0,006 \\
-0,027 \\
0,005 \\
0,055 \\
0,048 \\
0,044 \\
0,083 \\
0,040 \\
0,041 \\
0,012 \\
0,050 \\
0,062 \\
0,051 \\
0,042\end{array}$ & \\
\hline
\end{tabular}

Fonte: UNICEF6 (1991); World Bank? (1991) 


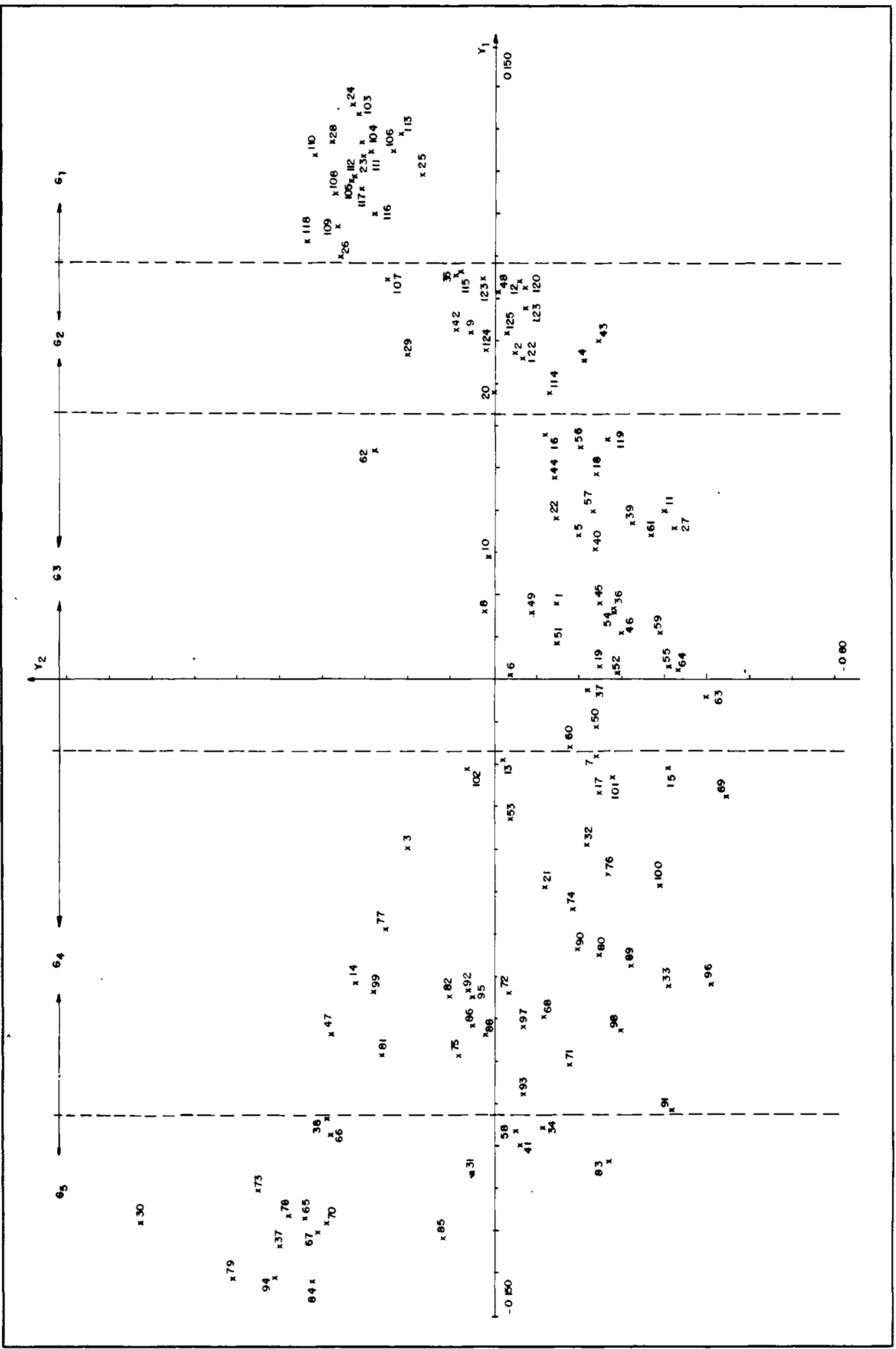

Figura 1. Ordenação dos países utilizando-se os dois primeiros componentes principais $\left(Y_{1}, Y_{2}\right)$. Indicação dos 5 grupos formados. 


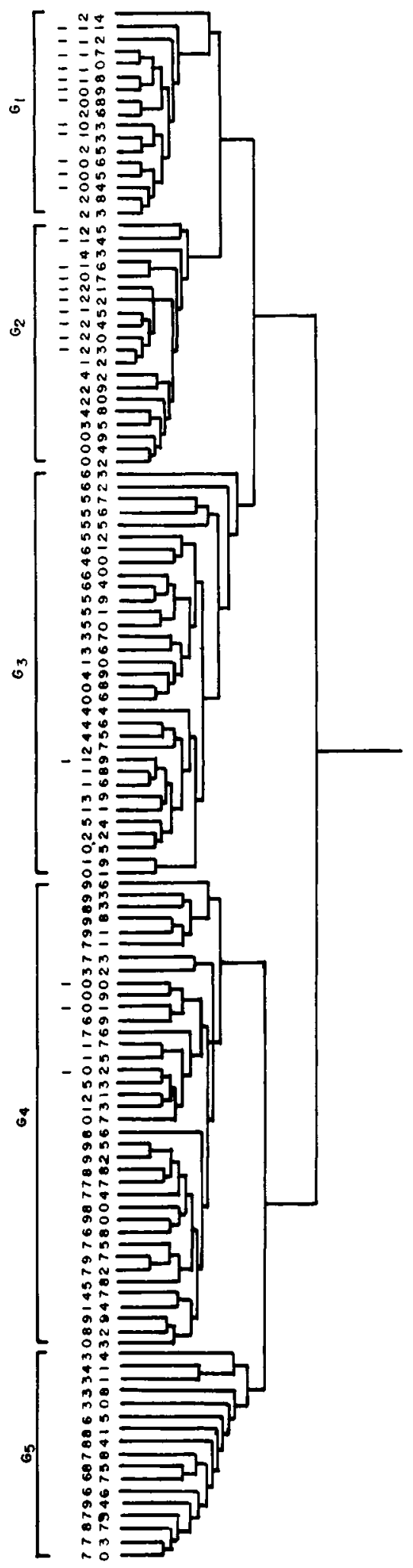

Figura 2. Dendrograma resultante da Análise de Agrupamento dos países, utilizando-se a Distância Euclidiana Média como coeficiente de similaridade e algorítmo UPGMA. lores de $Y_{1}$ (média de 0,120 e desvio de 0,010 ), como para valores de $Y_{2}$, todos positivos e relativamente grandes, em consequiência de $P N B, R A D$ e TV elevados.

A ordenação pelo primeiro componente principal (Tabela 2) situou o Brasil em $54^{2}$ lugar entre os 125 países estudados. Esta classificação do Brasil concordou com os resultados obtidos anteriormete 2,4 . Embora este tipo de enfoque, usando indicadores médios, possa não refletir particularidades dos países, é o possível de ser utilizado. No caso do Brasil, devido à sua dimensão territorial e a heterogeneidade entre suas regiōes, o país "médio" aqui estudado, na verdade, não reflete sua multiplicidade de características. Uma complementação interessante do presente estudo seria verificar o comportamento dos Estados e ou das regioes brasileiras face aos demais paises do Mundo. Os Estados das regioes Sul e Sudeste poderiam se localizar em G2 e os Estados das regið̄es Norte e Nordeste, talvez se localizassem em G4 ou G5.

A localização dos EUA em G1 era esperada ${ }^{2,4}$. Embora apresentando bolsões de pobreza, os indicadores médios dos EUA, principalmente aqueles de natureza econômica, devem ter sido responsáveis pelo valor máximo do primeiro componente entre todos os países. A mesma razão justifica a inclusão de países árabes como Kwait, Omã, Emirados Árabes Unidos e Arábia Saudita em G3.

Os antigos países comunistas da Europa se localizaram em G2, exceção feita a Albânia (G3). A China $\left(48^{\circ}\right)$ foi incluída em G3. Por outro lado, Cuba ficou na $23^{2}$ posição (em G2), em virtude de seus excelentes indicadores de saúde, embora seu PNB seja baixo (US\$ 1,170).

Dos países da América do Sul, Uruguai (29\%), Argentina $\left(32^{\circ}\right)$ e Chile (34) apresentaram é melhor desempenho, sendo incluídos em G2, o que confirmou os resultados obtidos anteriormente 2,4 . O pior desempenho ficou com a Bolívia $\left(83^{\circ}\right)$, localizada em G4. A inclusão de Trinidad-Tobago (36') em G2 deve ser destacada.

A Austrália $\left(11^{\circ}\right)$ e a Nova Zelândia $\left(18^{\circ}\right)$, junto com Japão $\left(5^{\circ}\right)$, Canadá $\left(8^{\circ}\right)$ e EUA $\left(1^{\circ}\right)$, foram os únicos países não europeus incluídos em G1.

Os grupos G4 e G5 incluíram a totalidade dos paises africanos.

Como os dois procedimentos usados (AA e ACP) não excluem uma certa subjetividade no processo de separação dos grupos ${ }^{1}$, o estudo foi completamentado com a Análise de Variância Multivariada (MAV). Esta análise, ao contrário das anteriores, tem como ponto de partida o conjunto dos 125 países divididos em 5 grupos. A comparação entre os mesmos para o conjunto das variáveis resultou significativa (distância generalizada de Mahalanobis: $\left.D^{2}=4287,4 ; p<0,001\right)$. Foi, tam- 
bém, significativa a diferença entre os grupos quando comparados dois a dois. Esses resultados mostraram que a separação dos países em 5 grupos foi coerente (heterogeneidade entre grupos).

Os paises foram reclassificados em um dos 5 grupos, calculando-se o valor da probabilidade associada à reclassificação, utilizando-se as funçбes discriminantes lineares de cada grupo, geradas no processo. Assim procedendo foi possivel afirmar que:

a) a taxa de erro de reclassificação em G1 foi de 5,6\% com único país deste grupo, a Espanha, reclassificada em G2. Dos demais paises corretamente reclassificados, a Nova Zelândia mostrou-se o menos típico de G1, com probabilidade 0,95 de reclassificação correta;

b) a taxa de erro de reclassificação em G2 foi nula. $\mathrm{O}$ país menos típico de $\mathrm{G} 2$ foi TrinidadTobago (com probabilidade igual a 0,87 );

c) para G3 a taxa de erro de reclassificação foi nula, sendo menos típicos deste grupo a Albânia e o Líbano;

d) em G4, também, foi nula a taxa de erro. Os países menos típicos deste grupo foram: Libéria (prob $=0,58$ ) e Zimbábue (prob $=0,80$ );

e) em G5 houve uma reclassificação errônea (taxa de erro igual a $5,6 \%$ ), com o Iêmen sendo reclassificado em G4.

Foram geradas duas variáveis canônicas, responsáveis por $94,5 \%$ da informação total fornecida pelo conjunto das 26 variáveis originais. A Figura 3 apresenta a localização dos países como pontos no plano das variáveis canônicas (CAN1, CAN2). Foram calculados os pontos médios dos grupos, indicados pelos escores centroídes das duas variáveis canônicas: G1 $(15,31 ;-2,33) ; \mathrm{G} 2$ $(9,78 ; 0,79) ; \mathrm{G} 3(1,83 ; 1,46) ; \mathrm{G} 4(-7,40 ; 0,48)$ e G5 $(-13,79 ;-2,17)$.

Conforme se depreende dos resultados apresentados, a concordância conseguida com os três métodos de análise multivariada empregados foi exce'ente.

$\mathrm{Na}$ Tabela 3 são apresentados os valcies médios das variáveis nos 5 grupos e a comparação destes para cada variável. Os grupos meñs separados foram G1 e G2 e, em menor esca! „, G4 e G5, com o G3 resultando significativamerte diferente (intermediário) para a maioria das variáveis. Mesmo assim, conforme já foi ressaltado, os grupos G1 e G2 diferiram para o conjunto das variáveis conseqüência, principalmente, da diferença apresentada para PNB, RAD, TV, M2GM e M2GF. Para TMM5, TMI e MAT, entre cutras; embora a diferença entre os dois grupos não tenha sido significativa ela deve ser ressaltada como sendo de relevância prática.

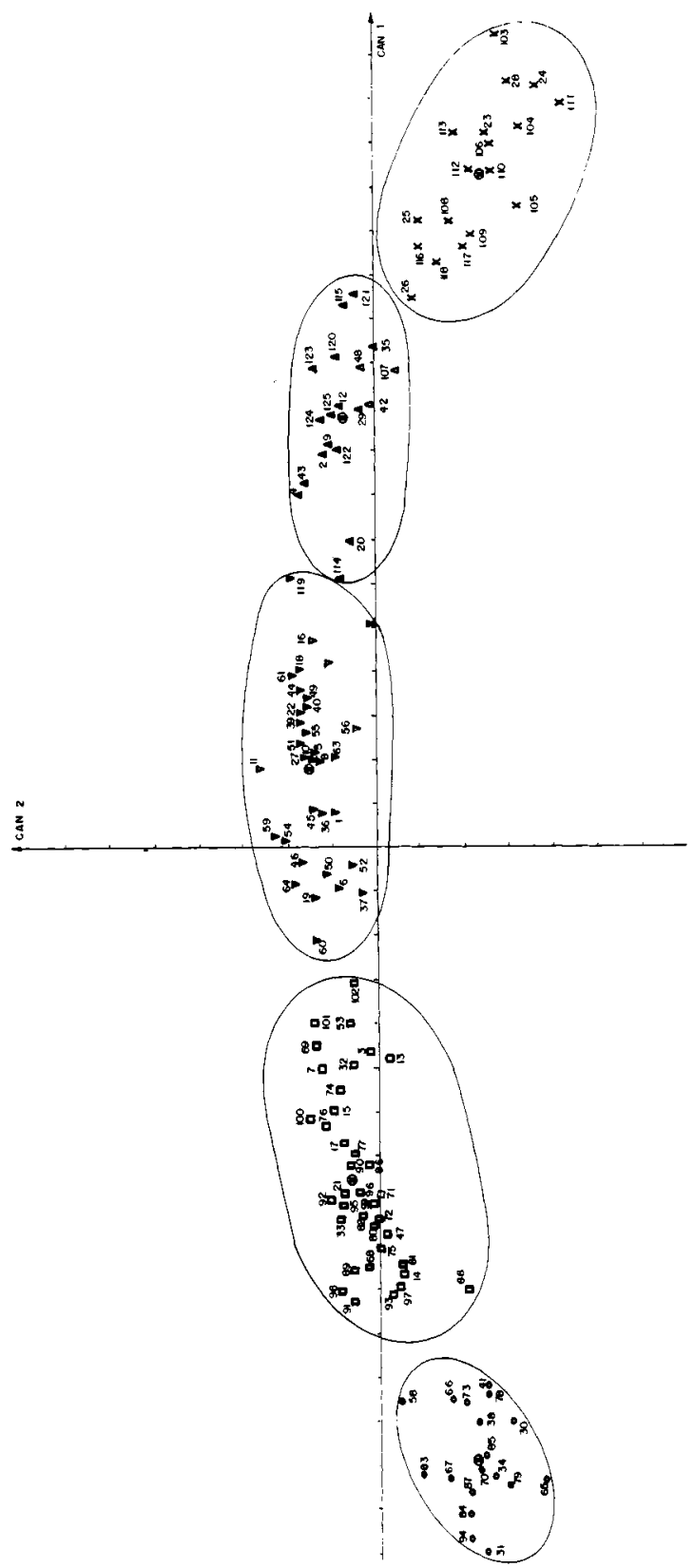

Flgura 3. Localização dos paises utilizando-se as duas primeiras variáveis canónicas (CAN 1, CAN 2) obtida na Análise de Variância Multivariada. Indicaçăo dos grupos con seus respectivos símbolos ( $0-G 1 ; \square-G 2 ; \nabla-G 3$; $\Delta-G 4 ; x-G 5) \in$ de seus pontos centrais (0) (Confira Tabela 2)

Pela Tabela 3 verificou-se que as taxas de imunização (DPT, POL, SAR) não diferiram em G1, G2 e G3, o que poderia ser explicado pelo fato dos países mais desenvolvidos não estarem promovendo programas intensos de vacinação, talvez em conseqüência da menor importância atribuída a pouca ocorrência de moléstias infecciosas. 
Tabela 3. Valores médios das variáveis originais nos 5 grupos de países inferidos na Análise de Agrupamento e na Análise de Componentes Principais. Diferença estatística entre os grupos para cada variável (resultado obtido na Análise de Variância Multivariada).

\begin{tabular}{|c|c|c|c|c|c|c|}
\hline Variável & G1 & G2 & $\begin{array}{c}\text { Grupo } \\
\text { G3 }\end{array}$ & G4 & G5 & Estatistica \\
\hline $\begin{array}{l}\text { EXP } \\
\text { ALF } \\
\text { NAT } \\
\text { FERT } \\
\text { LEIT } \\
\text { MED } \\
\text { ENF } \\
\text { TMM5 } \\
\text { TMI } \\
\text { RNBP } \\
\text { CAL } \\
\text { DPT } \\
\text { POL } \\
\text { SAR } \\
\text { NAS } \\
\text { MAT } \\
\text { PNB } \\
\text { N1GG } \\
\text { RAD } \\
\text { TV } \\
\text { C1G } \\
\text { M2GM } \\
\text { M2GF } \\
\text { CPOP } \\
\text { URB } \\
\text { CURB }\end{array}$ & $\begin{array}{c}76 \\
99 \\
12,8 \\
1,7 \\
120 \\
525 \\
160 \\
9 \\
8 \\
5 \\
130 \\
88 \\
91 \\
79 \\
99 \\
7 \\
18514 \\
104 \\
871 \\
461 \\
98 \\
93 \\
97 \\
0,4 \\
78 \\
0,7\end{array}$ & $\begin{array}{c}73 \\
93 \\
17,3 \\
2,2 \\
203 \\
756 \\
563 \\
21 \\
17 \\
7 \\
131 \\
89 \\
91 \\
89 \\
97 \\
38 \\
3901 \\
103 \\
423 \\
233 \\
92 \\
74 \\
79 \\
0,9 \\
70 \\
1,8\end{array}$ & $\begin{array}{c}67 \\
76 \\
30,8 \\
4,1 \\
608 \\
3223 \\
1898 \\
67 \\
45 \\
10 \\
116 \\
85 \\
88 \\
81 \\
77 \\
125 \\
2747 \\
105 \\
268 \\
124 \\
77 \\
58 \\
54 \\
2,6 \\
55 \\
4,0\end{array}$ & $\begin{array}{c}56 \\
54 \\
43,4 \\
6,1 \\
855 \\
14292 \\
3290 \\
138 \\
89 \\
15 \\
96 \\
67 \\
68 \\
65 \\
43 \\
420 \\
679 \\
78 \\
158 \\
23 \\
57 \\
27 \\
21 \\
3,0 \\
34 \\
5,2\end{array}$ & $\begin{array}{c}42 \\
32 \\
46,9 \\
6,5 \\
2572 \\
42123 \\
8866 \\
228 \\
136 \\
20 \\
89 \\
41 \\
41 \\
42 \\
19 \\
726 \\
278 \\
53 \\
83 \\
5 \\
44 \\
16 \\
8 \\
2,6 \\
21 \\
5,8\end{array}$ & $\begin{array}{l}(G 1=G 2)>G 3>G 4>G 5 \\
(G 1=G 2)>G 3>G 4>G 5 \\
(G 1=G 2)<G 3<(G 4=G 5) \\
(G 1=G 2)<G 3<(G 4=G 5) \\
(G 1=G 2=G 3=G 4)<G 5 \\
(G 1=G 2=G 3)<G 4<G 5 \\
(G 1=G 2)<G 5 \\
(G 1=G 2)<G 3<G 4<G 5 \\
(G 1=G 2)<G 3<G 4<G 5 \\
(G 1=G 2)<G 5 \\
(G 1=G 2=G 3)>(G 4=G 5) \\
(G 1=G 2=G 3)>G 4>G 5 \\
(G 1=G 2=G 3)>G 4>G 5 \\
(G 1=G 2=G 3)>G 4>G 5 \\
(G 1=G 2)>G 3>G 4>G 5 \\
(G 1=G 2)<(G 4=G 5) \\
G 1>(G 2=G 3)>(G 4=G 5) \\
(G 1=G 2=G 3)>G 4>G 5 \\
G 1>G 2>G 3>(G 4=G 5) \\
G 1>G 2>G 3>(G 4=G 5) \\
(G 1=G 2)>G 3>G 4>G 5 \\
G 1>G 2>G 3>G 4>G 5 \\
G 1>G 2>G 3>G 4>G 5 \\
(G 1=G 2)<G 3=G 4=G 5 \\
(G 1=G 2)>G 3>(G 4=G 5) \\
(G 1=G 2)<G 3<(G 4=G 5)\end{array}$ \\
\hline
\end{tabular}

Fonte: UNICEFB (1991); World Bank7 (1991).

Os precários indicadores educacionais, econômicos, culturais e de assistência à saúde nos grupos G4 e G5 foram acompanhados por expectativas de vida muito baixas e por taxas muito elevadas de mortalidade infantil e materna.

CURI, P. R. [Clustering of countries evaluated by standard of living indicators]. Rev. Saúde Pública, 27: 12734, 1993. The position of 125 countries is studied on the basis of a collection of 26 basic, health, economic and educational indicators. Multivariate statistical methods were used, including Cluster Analysis, Principal Component Analysis and Multivariate Analysis of Variance. The most discriminating variables were life expectancy the child mortality rate, the mortality rate of children of less than five years of age, the birth and fertility rates and the high-school female matriculation rate. The first principal component was interpreted as a measure of the living standard which made it possible to place the countries in order. Five clusters of countries are suggested.

Keywords: Quality of life. Cluster analysis. Discriminant analysis. Health statistics.

\section{Referências Bibliográficas}

1. CURI, P. R. Resultados comparativos de alguns métodos de análise multivariada aplicados a um conjunto de dados. Rev. Matem. Estat., 1: 55-7, 1983.

2. CURI. P. R.; CONCEIÇÃO, E. P.; FELÍCIO, E. Análise de componentes principais e de agrupamento na avaliaçảo demográfica de países. Salusvita, 9: 45-62, 1990.

3. CURI, P. R.; ALVES, A.; BECKERS, P. J, e TERADA, L. Anf́lise de agrupamento e de componentes principais na avaliação de tabelas multivariadas ( $\mathrm{n} \times \mathrm{p}$ ). Rev. Matem. Estat., 9: 35-49, 1991.

4. NUNES, O. Z. G. S.; SILVA, M. S.; CURI, P. R. Agrupamento de países segundo indicadores básicos relacionados à situação mundial da infância. Rev. Ciênc. Bioméd. 12: 89-104, 1991.

5. SNEATH, P. H. A. \& SOKAL, R. R. Numerical taxonomy. San Francisco, W. H. Freeman, 1973.

6. FUNDO DAS NAÇÓES UNIDAS PARA A INFÂNCIA (UNICEF). Siluaçăo mundial da infancia-1991. Brasília, 1991.

7. WORLD Development Report 1990. WASHINGTON, World Bank, 1991.

Recebido para publicaçäo em 13.7.1992

Reapresentado em 23.4.1993

Aprovado para publicaçấo em 26.4.1993 